\title{
Using close stars as probes of hot accretion flow in Sgr $A^{*}$
}

\author{
S. Nayakshin \\ Max-Planck-Institut für Astrophysik, Karl-Schwarzschild-Str.1, 85741 Garching, Germany \\ e-mail: serg@mpa-garching.mpg.de
}

Received 20 October 2004 / Accepted 6 November 2004

\begin{abstract}
The extremely hot and tenuous accretion flow in the immediate vicinity of Sgr A* is believed to be invisible (too dim) in the X-ray band, except for short X-ray flares. Here we point out that during pericenter passages, close brightest stars irradiate the inner region of the accretion flow, providing a plenty of optical/UV photons. These seed photons are Compton up-scattered by the hot electrons of the accretion flow to higher frequencies, some into the X-ray band, potentially making the innermost accretion flow much brighter in X-rays than usual. We propose to use coordinated near infra-red and X-ray observations of close star passages to put constraints onto Sgr $\mathrm{A}^{*}$ accretion theories. The absence of a noticeable change in the steady emission of Sgr A* as observed by Chandra in the year 2002, when the star named S2 passed through a pericenter of its orbit, already rules out the hotter of the "standard" Advection-Dominated Accretion Flows. The less dense accretion flows, in particular the model of Yuan et al. (2003), passes the test and is constrained to accretion rates no larger than $\sim$ few $\times 10^{-7} M_{\odot}$ year $^{-1}$.
\end{abstract}

Key words. accretion, accretion disks - black hole physics - Galaxy: center - radiation mechanisms: general

\section{Introduction}

Sgr $\mathrm{A}^{*}$ is the super massive black hole (SMBH) in the center of our Galaxy (Reid et al. 1999; Schödel et al. 2002; Ghez et al. 2003a). The gas from the winds of the surrounding young massive stars should be able to maintain the accretion rate on the SMBH at a sizable fraction of the Bondi accretion rate if the SMBH can accept the gas at this rate. The Bondi accretion rate is estimated to be $\sim 10^{-6} M_{\odot}$ year $^{-1}$ (Baganoff et al. 2003), whereas the linear polarization measurements at a range of radio wavelengths constrain the accretion rate onto the SMBH to a value of $\sim 10^{-8}-10^{-7} M_{\odot}$ year $^{-1}$ (e.g., Bower et al. 2003). The latter estimate is based on somewhat uncertain assumptions about the magnetic field equipartition and absence of many magnetic field reversals (Ruszkowski \& Begelman 2002).

It would certainly be beneficial to have another physically independent method to constrain the properties of the accretion flow near the event horizon. Unfortunately, in the X-ray band, it appears that the quiescent $\mathrm{Sgr} \mathrm{A}^{*}$ emission is resolved (Baganoff et al. 2003) and can be explained by thermal bremsstrahlung from the capture radius region (Quataert 2002). The inner flow may thus be simply invisible to the observer unless there is a sizable X-ray emission from a jet (e.g., Yuan et al. 2002), or during strong X-ray flares (Baganoff et al. 2001).

In this letter we note that comptonization of the radiation of the closest of the observed bright stars by the hot accretion flow may be used to this effect. These stars produce as much as $L_{*} \sim 10^{5} L_{\odot} \sim 4 \times 10^{38} \mathrm{erg} / \mathrm{s}$ in optical-UV radiation, the amount far greater than the total bolometric luminosity of $\mathrm{Sgr} \mathrm{A}^{*}, L_{\mathrm{t}} \sim 10^{36} \mathrm{erg} / \mathrm{s}$. In a single Compton scattering, the photon energy on average increases by a factor $\gamma^{2}$, where $\gamma$ is the Lorenz $\gamma$-factor of the electron taking part in the up-scattering. Averaged over a relativistic Maxwellian electron distribution with temperature $T_{\mathrm{e}}$, the average boost factor becomes $16\left(k_{\mathrm{B}} T_{\mathrm{e}} / m_{\mathrm{e}} c^{2}\right)^{2}$, where $k_{\mathrm{B}}$ is the Boltzmann constant, and $m_{\mathrm{e}}$ is the electron mass. The typical frequency of a photon emitted by the star is around $v=3 k_{\mathrm{B}} T_{*} / h \approx 2 \times 10^{15} \mathrm{~Hz}$, where $T_{*}=30000$ Kelvin is the stellar temperature, and $h$ is the Planck constant. Chandra frequency band is centered around $v \sim 10^{18} \mathrm{~Hz}$. Therefore electrons with temperature of $T_{\mathrm{e}} \approx 3 \times 10^{10} \mathrm{~K}$ are the most efficient in scattering of S2 radiation into the Chandra band.

Such high temperature electrons are present in both the canonical ADAF (without winds) model, e.g. Narayan (2002), and even more so in the less dense but hotter Non-Radiative Accretion Flows (NRAFs) that incorporate effects of gas outflows. Since $\mathrm{Sgr} \mathrm{A}^{*}$ is very dim in X-rays in its normal "quiescent" state, e.g. $L_{\mathrm{X}} \sim 10^{33} \mathrm{erg} \mathrm{s}^{-1}$, there is then a hope of detecting a comptonized "echo" of a close star passage.

\section{Analytical estimates}

We shall rely on the accretion flow models by Narayan (2002) and Yuan et al. (2003), who used $M_{\mathrm{BH}}=2.6 \times 10^{6} M_{\odot}$. Recently, Sgr A* mass has been determined to be larger, e.g. $M_{\mathrm{BH}}=(3-4) \times 10^{6} M_{\odot}($ Genzel et al. 2003; Ghez et al. 2003a). We thus use an intermediate value of $M_{\mathrm{BH}}=3 \times 10^{6} M_{\odot}$.

The Thomson optical depth, $\tau_{\mathrm{T}}$, of the hot accretion flow in Sgr A* is quite small: $\tau_{\mathrm{T}} \sim 10^{-3} n_{8} r_{1}$, where $n_{8}=n_{\mathrm{e}} / 10^{8}$ is the typical electron density in units of $10^{8} \mathrm{~cm}^{-3}$, and 
$r_{1}=R / 10 R_{\mathrm{S}}$. (Note that further out the optical depth of the flow is even smaller.) Therefore, only the first order scattering of the stellar photons need be considered.

At the moment of the closest approach to the $\mathrm{SMBH}$, the star S2 in particular, was $D=R_{\mathrm{p}} \sim 2000 R_{\mathrm{S}}$ away from $\mathrm{Sgr} \mathrm{A}^{*}$, where $R_{\mathrm{S}}=2 G M_{\mathrm{BH}} / c^{2}$ is the Schwarzschild radius of the $\mathrm{SMBH}, D$ is the current distance between the star and Sgr $\mathrm{A}^{*}$, and $R_{\mathrm{p}}$ is the pericenter distance of the orbit. The radiation energy density near the SMBH is thus

$u_{\mathrm{rad}}=\frac{L_{*}}{4 \pi D^{2} c} \approx 10^{-3} \frac{L_{5}}{D_{2000}^{2}} \mathrm{erg} \mathrm{s}^{-1}$,

where $L_{5}=L_{*} / 10^{5} L_{\odot}, D_{2000}=D /\left(2000 R_{\mathrm{S}}\right)$.

We now introduce $N_{\text {he }}$ as the total number of electrons in the hot accretion flow that are able to up-scatter an optical/UV photon with frequency $v_{*} \simeq 2 \times 10^{15} \mathrm{~Hz}$ into the Chandra band, $v_{\mathrm{x}} \simeq 10^{18} \mathrm{~Hz}$. The corresponding mass of the accretion flow ions, assumed to be all protons for simplicity, is clearly $\tilde{M}_{\text {acc }} \approx$ $m_{\mathrm{p}} N_{\text {he }}$, where $m_{\mathrm{p}}$ is the proton mass. Since scattering conserves number of photons, we can estimate the X-ray luminosity of the up-comptonized $\mathrm{S} 2$ emission as

$L_{\mathrm{x}} \sim N_{\mathrm{he}} u_{\mathrm{rad}} c \sigma_{\mathrm{T}} \frac{v_{\mathrm{x}}}{v_{*}} \approx 10^{33} \frac{M_{-10} L_{5}}{D_{2000}^{2}} \mathrm{erg} \mathrm{s}^{-1}$,

where $M_{-10} \equiv \tilde{M}_{\text {acc }} / 10^{-10} M_{\odot}$. Recall that quiescent X-ray luminosity of $\mathrm{Sgr} \mathrm{A}^{*}$ is $L_{\mathrm{x}} \simeq 2 \times 10^{33} \mathrm{erg} / \mathrm{s}$. Thus, if the region of accretion flow with $T_{\mathrm{e}} \sim 3 \times 10^{10} \mathrm{~K}$ had a mass of around $\tilde{M}_{\text {acc }}=10^{-10} M_{\odot}$ or larger, the up-comptonized S2 emission would have been measurable in 2002 by Chandra.

We can relate the accretion flow mass $M_{\text {acc }}$ at a given dimensionless radius $r$ to the accretion rate as

$M_{\mathrm{acc}} \sim \dot{M} t_{\text {visc }}$

where $t_{\text {visc }}$ is the viscous time estimated in $\alpha$-viscosity accretion flows as

$t_{\mathrm{visc}} \simeq t_{\mathrm{dyn}} \alpha^{-1} \frac{R^{2}}{H^{2}} \simeq 30 \mathrm{~s} r^{3 / 2} \alpha^{-1} \frac{R^{2}}{H^{2}}$,

where $t_{\text {dyn }}=R / v_{\mathrm{K}}$ is the local dynamical time and $H$ is the geometrical thickness of the accretion flow. For flows considered here $H / R \sim 1$. For example, with an accretion rate of $\dot{M}=10^{-7} \dot{M}_{-7} M_{\odot}$ year $^{-1}$, we have

$M_{\mathrm{acc}} \simeq 3 \times 10^{-11} M_{\odot} \dot{M}_{-7} \alpha_{-1}^{-1} r_{1}^{3 / 2}$,

where $\alpha_{-1}=\alpha / 0.1$. Requiring $M_{\text {acc }} \lesssim 10^{-10} M_{\odot}$, we have

$\dot{M} \lesssim 3 \times 10^{-7} \alpha_{-1} r_{1}^{3 / 2}$.

This is a realistic range of accretion rates for Sgr $\mathrm{A}^{*}$ (Narayan 2002; Yuan et al. 2003).

\section{Calculation of the comptonized spectrum}

The estimates above are useful but nonetheless a more careful calculation is required as an accretion flow spans a range of temperatures and its density profile may be changing in a non-trivial way due to, e.g., outflows. For definitiveness, we

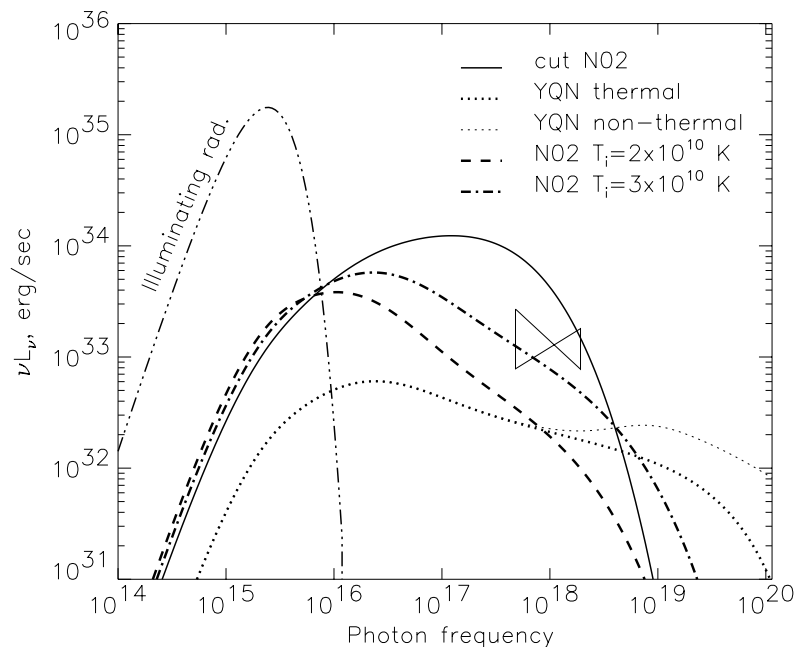

Fig. 1. The up-comptonized emission of the close star S2 during its closest approach to Sgr $\mathrm{A}^{*}$ in 2002 for five different accretion flows, as marked in the figure. The spectrum of the star illuminating the inner 100 Schwarzschild radii of the flow is also shown for comparison. The Chandra data with uncertainties (Baganoff et al. 2003) are shown with the bow-tie.

shall use the accretion flow structure as available in models of Narayan (2002) and Yuan et al. (2003).

The electron temperature is at most equal to the local virial temperature, e.g. temperature at which the thermal energy of the gas is equal to the gravitational potential energy: $T_{\mathrm{vir}}=$ $(2 / 3) G M_{\mathrm{BH}} m_{\mathrm{p}} /\left(k_{\mathrm{B}} R\right)=3.6 \times 10^{12} r^{-1} \mathrm{~K}$. Thus, $T_{\mathrm{e}} \leq 4 \times 10^{9} \mathrm{~K}$ for $r \geq 10^{3}$. Accordingly, we do not consider Compton scattering in the outer, $r \geq 10^{3}$, regions of the flow. In practice $T_{\mathrm{e}}$ is still smaller than $T_{\text {vir }}$, and hence it is only the inner few hundred Schwarzschild radii of the flow that are important. Since these radii are much smaller than $\mathrm{S} 2$ pericenter distance, $R_{\mathrm{p}} \sim 2000 R_{\mathrm{S}}$, we can safely assume that $u_{\mathrm{rad}}=$ const. in this optically thin region.

The emitted comptonized spectrum is calculated as

$L_{v}=c \int_{R_{\mathrm{i}}}^{R_{\text {out }}} \mathrm{d} V n_{\mathrm{e}}(R) \int_{0}^{\infty} \mathrm{d} v_{1} \frac{\mathrm{d} u_{\mathrm{rad}}\left(v_{1}\right)}{\mathrm{d} \nu_{1}} \sigma\left(v, v_{1}, T_{\mathrm{e}}\right)$,

where $\mathrm{d} V=4 \pi R^{2} \mathrm{~d} R$ is the volume element, $v_{1}$ is the frequency of the photon before scattering and $v$ is that after the scattering, and $\sigma\left(v, v_{1}, T_{\mathrm{e}}\right)$ is the cross section for such a scattering averaged over the electron distribution function with temperature $T_{\mathrm{e}}$. The cross section is taken from Nagirner \& Poutanen (1994). We assume also that the bolometric luminosity of S2 is $L_{*}=10^{5} L_{\odot}$ and the spectrum is that of a blackbody at temperature $T=30000$ Kelvin.

Figure 1 shows several examples of the expected upcomptonized spectrum calculated for several different accretion flow models. The dash-multiple-dot curve labelled by "Illuminating rad". is the stellar radiation incident on the inner $100 R_{\mathrm{S}}$ sphere, plotted as a reference scale. The bow-tie is the Chandra data on emission of Sgr A* with the spectral uncertainty (Baganoff et al. 2003).

The solid curve is the simplest "cut-off" ADAF model used by Narayan (2002) to illustrate the need for a two-temperature nature of the accretion flow in Sgr A*. In this model the electron 
temperature at large radii follows the virial gas temperature but is capped at $T_{\mathrm{e}}=10^{10} \mathrm{~K}$ at smaller radii. We assumed $\alpha_{-1}=1$ and accretion rate of $\dot{M}=3 \times 10^{-6} M_{\odot}$ year $^{-1}$, the lower of the values used by Narayan (2002) (see his Eq. (3)). The model clearly over-predicts the emission in the X-ray band. This occurs despite the fact that electrons never reach the temperature of $3 \times 10^{10} \mathrm{~K}$. The electrons in the tail of the Maxwellian distribution produce the Chandra band X-rays, and since the accretion rate is an order of magnitude higher than the critical one estimated in Eq. (6), the model manages to emit a measurable $\mathrm{X}$-ray flux.

Next we test the more refined ADAF models by Narayan (2002). These employ a self-consistent description of the electron-ion Coulomb energy exchange rather than the simple temperature cutoff. In particular, we approximate the electron temperature profiles in his Fig. 5 as

$T_{\mathrm{e}}= \begin{cases}T_{0}[r / 3]^{-1 / 2}, & \text { if } T_{\mathrm{e}}<T_{\mathrm{vir}}(r) \\ T_{\mathrm{vir}}(r), & \text { otherwise, }\end{cases}$

where $T_{0}$ is the electron temperature at $r=3$. In particular, we consider two values of $T_{0}$ that are approximately consistent with Fig. 5 of Narayan (2002): $T_{0}=2 \times 10^{10}$ and $T_{0}=3 \times 10^{10}$. Both models are for $\dot{M}=10^{-6} M_{\odot}$ year $^{-1}$ and $\alpha=0.1$. The higher initial electron temperature model is probably ruled out by the existing Chandra data on Sgr A* that show no noticeable variation in the X-ray flux during 2002 (Baganoff 2004, private communication). The lower temperature model may be however below what could have been detected by Chandra.

Finally, we also test the more recent and more physically complete NRAF models for Sgr A* by Yuan et al. (2003). Their Fig. 2 shows the electron temperature and the density profiles for one particular set of parameters that fit $\mathrm{Sgr} \mathrm{A}^{*}$ broad band spectrum quite well. Due to strong thermally driven outflows, the radial profile of density is $\rho(r) \propto r^{-3 / 2+s}$, with $s=0.27$. Note that for ADAFs $s=0$. The temperature profile in Yuan et al. (2003) is quite close to a power-law with $T(r) \propto r^{-1+s} \simeq$ $r^{-3 / 4}$. The accretion rate close to the SMBH in this model is $\dot{M}\left(3 R_{\mathrm{S}}\right)=4 \times 10^{-8} M_{\odot}$ year $^{-1}$.

Yuan et al. (2003) also include a non-thermal component in the electron distribution. As we find that the latter does not produce a dominant contribution to the up-comptonized S2 emission in the Chandra band, and since we do not have access to the exact model by Yuan et al. (2003), we set the minimum and maximum $\gamma$-factors as following: $\gamma_{\min }=30$ and $\gamma_{\max }=2000$, in a rough agreement with Fig. 2 of Yuan et al. (2003).

Figure 1 shows the resulting comptonized spectrum (fine dotted curves) at the pericenter of S2 orbit for the Yuan et al. (2003) model. The higher of the curves includes both thermal and non-thermal electrons, whereas the other one includes scattering due to the thermal electrons only. In both cases the resulting X-ray emission is about a factor of 10 below the quiescent X-ray emission of Sgr $\mathrm{A}^{*}$, so that the model of Yuan et al. (2003) clearly passes the test.

\section{Lightcurve of the event}

The trajectory of $\mathrm{S} 2$ is calculated based on the best fit to its orbit by Ghez et al. (2003a). The background optical-UV radiation
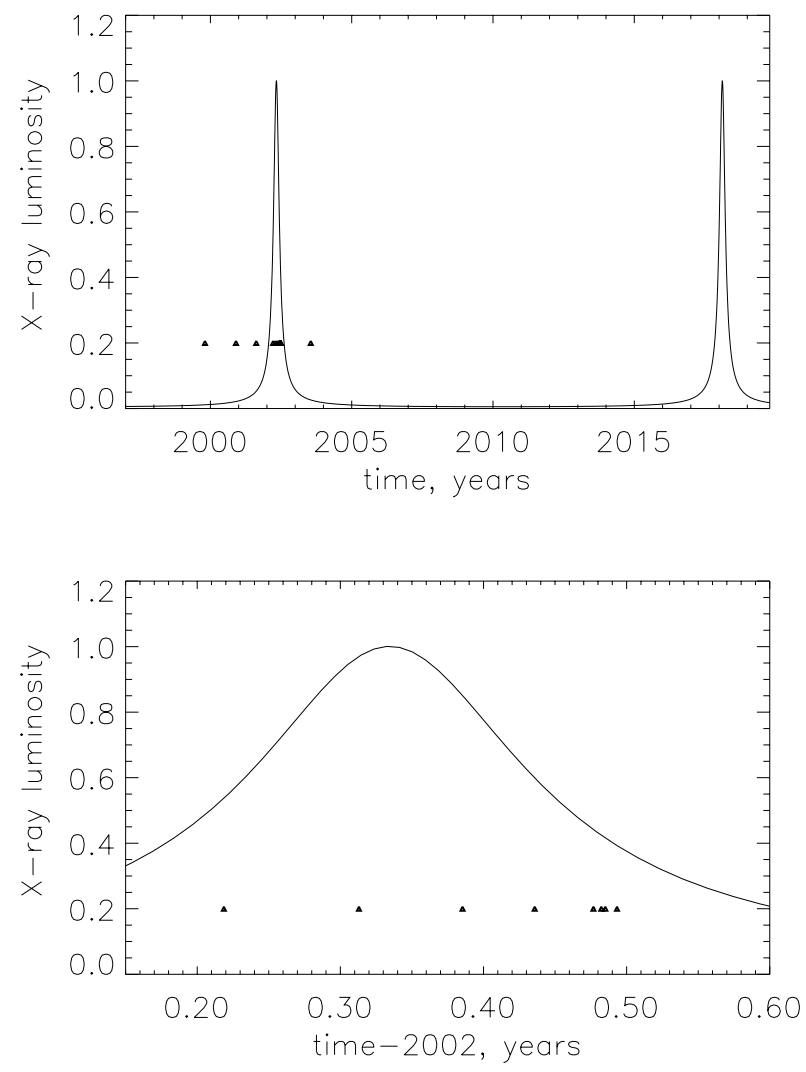

Fig. 2. The lightcurve of the optical/UV radiation of the close star S2 up-comptonized by the accretion flow into the X-ray band. The upper panel shows the lightcurve on time scales of tens of years, while the lower one shows the emission during 2002 when S2 passes through the pericenter of its orbit. The filled triangles mark the epochs when Chandra observed the Galactic Center region (Park et al. 2004; Eckart et al. 2004). The $y$-coordinate of the triangles in the figure is arbitrary.

energy density near $\operatorname{Sgr} \mathrm{A}^{*}$ location is assumed to be dominated by the whole of the $\mathrm{Sgr} \mathrm{A}^{*}$ star cluster, whose luminosity is of order $L_{\mathrm{cl}}=10^{7} L_{\odot}$, and whose size is $R_{\mathrm{cl}} \simeq 0.2$ parsec (Genzel et al. 2003). Thus, $u_{\mathrm{bg}}=L_{\mathrm{cl}} / 4 \pi R_{\mathrm{cl}}^{2}$. We assume a spectrum identical to that of S2 for simplicity. The background is quite negligible in comparison with the radiation energy density $u_{\text {rad }}$ of S2 during its pericenter passage (Eq. (1)).

Since the cooling time of the hot electrons due to inverse Compton effect is very long compared to the local dynamical time (Chang \& Choi 2003), it can be neglected. The X-ray lightcurve is then

$L_{\mathrm{x}}=L_{\mathrm{x}, \mathrm{p}}\left[\frac{R_{\mathrm{p}}}{D}\right]^{2}$,

where $D$ is the current distance between the SMBH and $\mathrm{S} 2$. The resulting $\mathrm{X}$-ray lightcurve, normalized to the maximum reached at the pericenter, $L_{\mathrm{x}, \mathrm{p}}$, is plotted in Fig. 2 . The upper panel shows the lightcurve on the scales of tens of years, whereas the lower panel concentrates on that during the year 2002 only. Clearly the duration of the event is several months. Very luckily, Chandra observed Sgr A* (filled triangles in Fig. 2) on many occasions in 2002 (e.g. see Park et al. 2004). It is thus possible that a careful analysis of Chandra 
2002 data would be able to constrain the amount of an extra up-comptonized X-ray flux to a small fraction of the quiescent $\mathrm{X}$-ray luminosity of Sgr A* Such an analysis may be facilitated by the fact that the lightcurve of the event is well determined due to small overall errors in the trajectory of the star S2 (Schödel et al. 2002; Ghez et al. 2003b).

\section{Discussion}

We have shown that up-comptonization of the optical/UV radiation of the star S2, which famously passed through the pericenter of its orbit only 2000 Schwarzschild radii away from Sgr A* (Schödel et al. 2002), constrains the properties of the accretion flow in the immediate vicinity of the SMBH. These constraints are completely independent from those due to the rotation measure limits in the radio wavelengths (Bower et al. 2003), and are thus complimentary to those results. We urge the observers to search the Chandra 2002 data for signatures of the transient brightening with the lightcurve shown in Fig. 2. Such an analysis may perhaps yield much tighter constraints on the amplitude of the effect than Fig. 1 indicates.

Future observations of close star passages may be more constraining that that from the year 2002 if (i) the star approaching the SMBH is brighter and/or closer to Sgr $\mathrm{A}^{*}$ than $2000 R_{\mathrm{S}}$; (ii) if more sensitive and well planned X-ray observations of the close stellar passage are made. In addition, interaction of stellar winds with the surrounding hot accretion flow may yield observable signatures in a range of wavelengths depending on the stellar mass loss rate and the outflow velocity and (unfortunately) not well constrained details of presumably collisionless wind/accretion flow shocks (e.g., see Loeb 2004).

The idea of using the close star passages to constrain the properties of the accretion flow around $\mathrm{Sgr} \mathrm{A}^{*}$ is not new. Chang \& Choi (2003) noted that the inverse Compton effect will provide an additional cooling of the hot electrons. However the corresponding relative change in the electron temperature is only $\lesssim 10^{-4}$ and appears very hard to observe in any wavelength. Here we showed that the up-comptonized $\mathrm{S} 2$ radiation itself, on the over hand, is much easier to observe since Sgr A* quiescent X-ray luminosity is very small. In addition, Nayakshin \& Sunyaev (2003) and Cuadra et al. (2003) have shown that passages of close stars could also be used to constrain the properties of a cold geometrically thin "inactive" accretion disk around Sgr $\mathrm{A}^{*}$. The fact that no near infra-red "echoes" or stellar eclipses were observed up to date probably indicates that such a disk does not presently exist in Sgr A*.

Concluding, we note that close stellar passages are of significant value for solving the riddle of $\mathrm{Sgr} \mathrm{A}^{*}$ and collisionless gas accretion onto SMBHs in general through a variety of physical processes.

Acknowledgements. The author acknowledges useful discussions with Fred Baganoff and Henk Spruit.

\section{References}

Baganoff, F. K., Bautz, M. W., Brandt, W. N., et al. 2001, Nature, 413, 45

Baganoff, F. K., Maeda, Y., Morris, M., et al. 2003, ApJ, 591, 891

Bower, G. C., Wright, M. C. H., Falcke, H., \& Backer, D. C. 2003, ApJ, 588, 331

Chang, H., \& Choi, C. 2003, A\&A, 410, 519

Cuadra, J., Nayakshin, S., \& Sunyaev, R. 2003, A\&A, 411, 405

Eckart, A., Baganoff, F. K., Morris, M., et al. 2004, ArXiv Astrophysics e-prints

Genzel, R., Schödel, R., Ott, T., et al. 2003, ApJ, 594, 812

Ghez, A. M., Becklin, E., Duchêne, G., et al. 2003a, Astron. Nachr., Vol. 324, No. S1, Special Suppl., The central 300 parsec of the Milky Way, ed. A. Cotera, H. Falcke, T. R. Geballe, \& S. Markoff [arXiv:astro-ph/0303151]

Ghez, A. M., Duchêne, G., Matthews, K., et al. 2003b, ApJ, 586, L127

Loeb, A. 2004, MNRAS, 350, 725

Nagirner, D. I., \& Poutanen, J. 1994, Single Compton scattering (Amsterdam: Harwood Academic Publishers)

Narayan, R. 2002, in Lighthouses of the universe: the most luminous celestial objects and their use for cosmology, ed. M. Gilfanov, R. Sunyaev, \& E. Churazov (Berlin: Springer), 405

Nayakshin, S., \& Sunyaev, R. 2003, MNRAS, 343, L15

Park, S., Muno, M. P., Baganoff, F. K., et al. 2004, ApJ, 603, 548

Quataert, E. 2002, ApJ, 575, 855

Reid, M. J., Readhead, A. C. S., Vermeulen, R. C., \& Treuhaft, R. N. 1999, ApJ, 524, 816

Ruszkowski, M., \& Begelman, M. C. 2002, ApJ, 573, 485

Schödel, R., Ott, T., Genzel, R., et al. 2002, Nature, 419, 694

Yuan, F., Markoff, S., \& Falcke, H. 2002, A\&A, 383, 854

Yuan, F., Quataert, E., \& Narayan, R. 2003, ApJ, 598, 301 